\title{
Control of Robot Manipulators Using Time-Delay Estimation and Fuzzy Logic Systems
}

\author{
Hyo-Jeong Bae*, Maolin Jin ${ }^{\dagger}$, Jinho Suh**, Jun Young Lee ***, Pyung-Hun Chang*** \\ and Doo-sung Ahn*
}

\begin{abstract}
A highly accurate model-free controller is proposed for trajectory tracking control of robot manipulators. The proposed controller incorporates time-delay estimation (TDE) to estimate and cancel continuous nonlinearities of robot dynamics, and exploits fuzzy logic systems to suppress the effect of the TDE error, which is due to discontinuous nonlinearities such as friction. To this end, integral sliding mode is defined using desired error dynamics, and a Mamdani-type fuzzy inference system is constructed. As a result, the proposed controller achieves the desired error dynamics well. Implementation of the proposed controller is easy because the design of the controller is intuitive and straightforward, and calculations of the complex robot dynamics are not required. The tracking performance of the proposed controller is verified experimentally using a 3-degree of freedom PUMAtype robot manipulator.
\end{abstract}

Keywords: Robot Manipulators, Time-Delay Estimation (TDE), Fuzzy Logic System (FLS), TimeDelay Control (TDC), Model-free control

\section{Introduction}

Robot manipulators are highly nonlinear, coupled, complex systems $[1,2]$. The tracking control of robot manipulators is one of the most challenging problems for control engineers. Accurate robot dynamic model is required to realize high accuracy-tracking control with model-based controllers [3-5]. The calculations of nonlinear robot models are complex to compute correctly, and even impossible if precise models of the robot manipulators are unknown. Therefore, it is difficult to control using conventional model-based techniques.

To realize model-free control, time-delay estimation (TDE) technique was proposed for control of robot manipulators in the late 1980s [6-8]. The TDE technique assumes that the unknown nonlinear function does not change much for a sufficiently small time period, and estimation and cancelation are realized through the TDE by judiciously using time-delayed information of the state derivatives and control inputs. The so-called time-delay control (TDC) for robot manipulators was proposed with this technique $[9,10]$. The key concept of the TDC is canceling unknown nonlinear dynamics of robot and injecting the desired error dynamics.

The TDE technique is simple, efficient, and yet effective.

$\dagger$ Corresponding Author: Korea Institute of Robot and Convergence, Korea.(mulimkim@kiro.re.kr)

* Dept. of Mechanical Design Engineering, Pukyong National University, Korea.

** Korea Institute of Robot and Convergence (KIRO), Korea

*** Dept. of Robotics Engineering, Daegu-Gyeongbuk Institute of Science and Technology (DGIST), Korea.

Received: October 8, 2016; Accepted: January 11, 2017
It has been applied to many plants: a permanent-magnet synchronous motor [11], a DC-DC boost converter [12], shape memory alloy actuators $[13,14]$, and chaotic systems $[15,16]$. Particularly, it has been also implemented for robot manipulators to perform trajectory tracking control, force control, impedance control, and dual arm cooperative control [17-21].

The TDC for robot manipulators consists of two elements $[9,10]$ : the robot dynamics canceling element using the TDE, and the desired error dynamics injecting element. Mostly, the TDE is computed from one-sample time delayed information. The TDE functions perfectly for continuous nonlinearities (such as gravity, Coriolis and centrifugal torques, viscous friction, disturbance) in robot dynamics, however, pulse type TDE error occurs for estimation of discontinuous nonlinearities such as Coulomb friction and Stiction $[19,22]$. The performance of the closed-loop systems are greatly affected by the TDE error.

To suppress the TDE error, a third element has been introduced, which includes ideal velocity feedback (IVF) $[19,21]$ and terminal sliding mode (TSM) [22]. The combination use of the TDE and TSM for robot control improves tracking performance of the TDC drastically [22]. The TDE-TSM [22] is a superset of the Hsia's formulation [10], and the controller using the TDE and IVF (TDE-IVF) [19]. When the TSM is activated by fractional power, the TDE-TSM provides faster convergence than the TDE-IVF due to the terminal sliding mode.

The TDE-TSM is not flawless. To implement terminal sliding mode, fractional power function pow $(\mathrm{x}, \gamma)$, where $0<\gamma<1$, is required to calculate. The calculation speed of fractional power function pow $(\mathrm{x}, \gamma)$ is dependent on 
operating systems (Windows, Linux, OS for embedded system), libraries versions, or languages $(\mathrm{C}, \mathrm{C}++$, Java, or MATLAB Simulink) [23, 24]. The calculation time is also heavily dependent on the input parameters. For example, the power function sometimes runs over 8,000 times slower than normal case. In the worst-case scenario, it takes dozens of millisecond to calculate the power function. Therefore, many users have developed approximate power function instead of exact power function, as a temporary solution [25-27]. The other shortcoming of the TDE-TSM is the control input chattering, which is highly undesirable. Because the slope of the TSM function is nearly 90 degree in the vicinity of equilibrium point when $0<\gamma<1$, control input chattering is invoked by the signum-like function near the equilibrium point.

To realize highly accurate tracking control of robot manipulator and to provide chattering reduction near sliding surface, in this paper, we propose a controller formulation using TDE and fuzzy logic systems. A Mamdani type fuzzy inference system (FIS) [28-30] is constructed by using commonsense rules that refer to indefinite quantities. To author's knowledge, this is the first paper that combines both the TDE technique and the intelligent technique (such as fuzzy logic systems) together to cope with the discontinuous nonlinearities for robot manipulators. The proposed controller consists of three elements: a TDE element, a desired error dynamic injection element, and a fuzzy logic compensator (FLC) to suppress the TDE error. Implementation of the proposed controller is easy because the design of the controller is intuitive and straightforward, and calculations of complex robot dynamics are not required. The proposed controller achieves the desired error dynamics well. The proposed controller has shown the better tracking performance compared with several TDE based controllers. The proposed controller is highly intuitive, efficient, and accurate; thus, it is a good candidate for practical use.

This paper is organized as follows. The next section proposes a high-accuracy tracking control of robot manipulators using the TDE and the FLC. In the third section, the proposed controller is compared experimentally with several TDE based controllers using a PUMA-type robot manipulator. Finally, conclusions are given.

\section{Proposed Control Law for Robot Manipulators}

\subsection{TDC for robot manipulators - Hsia's formulation}

$$
\mathbf{M}(\mathbf{q}) \ddot{\mathbf{q}}+\mathbf{C}(\mathbf{q}, \dot{\mathbf{q}}) \dot{\mathbf{q}}+\mathbf{G}(\mathbf{q})+\mathbf{F}(\mathbf{q}, \dot{\mathbf{q}})+\boldsymbol{\tau}_{d}=\boldsymbol{\tau},
$$

where $\mathbf{q}, \dot{\mathbf{q}}, \ddot{\mathbf{q}} \in \mathfrak{R}^{n}$ represent the position, velocity, and acceleration of the joints, respectively, and $\mathbf{M}(\mathbf{q}) \in \mathfrak{R}^{n \times n}$ stands for the generalized inertia matrix, $\mathbf{C}(\mathbf{q}, \dot{\mathbf{q}}) \in \mathfrak{R}^{n \times n}$ the Coriolis/centripetal matrix, $\mathbf{G}(\mathbf{q}) \in \mathfrak{R}^{n}$ the gravitational vector, $\mathbf{F}(\mathbf{q}, \dot{\mathbf{q}}) \in \mathfrak{R}^{n}$ the friction forces, $\boldsymbol{\tau}_{d} \in \mathfrak{R}^{n}$ the disturbance torques, and $\tau \in \Re^{n}$ the joint torques.

Introducing a positive diagonal matrix, $\overline{\mathbf{M}}$, one can obtain another expression of (1) as follows:

$$
\overline{\mathbf{M}} \ddot{\mathbf{q}}+\mathbf{N}(\mathbf{q}, \dot{\mathbf{q}}, \ddot{\mathbf{q}})=\tau
$$

where

$$
\mathbf{N}(\mathbf{q}, \dot{\mathbf{q}}, \ddot{\mathbf{q}})=[\mathbf{M}(\mathbf{q})-\overline{\mathbf{M}}] \ddot{\mathbf{q}}+\mathbf{C}(\mathbf{q}, \dot{\mathbf{q}}) \dot{\mathbf{q}}+\mathbf{G}(\mathbf{q})+\mathbf{F}(\mathbf{q}, \dot{\mathbf{q}})+\boldsymbol{\tau}_{d}
$$

The control objective is to make a robot position vector $\mathbf{q}$ follow the reference input trajectory $\mathbf{q}_{d}$. To this end, we first define $\mathbf{e}=\mathbf{q}_{d}-\mathbf{q}, \quad \dot{\mathbf{e}}=\dot{\mathbf{q}}_{d}-\dot{\mathbf{q}}$, and $\ddot{\mathbf{e}}=\ddot{\mathbf{q}}_{d}-\ddot{\mathbf{q}}$. The desired error dynamics is defined by

$$
\ddot{\mathbf{e}}+\mathbf{K}_{D} \dot{\mathbf{e}}+\mathbf{K}_{P} \mathbf{e}=\mathbf{0},
$$

where $\mathbf{K}_{D} \in \mathfrak{R}^{n \times n}$, and $\mathbf{K}_{P} \in \mathfrak{R}^{n \times n}$ are constant diagonal gain matrices. $\mathbf{K}_{D}$ is a derivative gain, and $\mathbf{K}_{P}$ is a proportional gain.

The control input can be selected as

$$
\tau=\overline{\mathbf{M}} \mathbf{u}_{0}+\hat{\mathbf{N}}(\mathbf{q}, \dot{\mathbf{q}}, \ddot{\mathbf{q}})
$$

where

$$
\mathbf{u}_{0}=\ddot{\mathbf{q}}_{d}+\mathbf{K}_{D} \dot{\mathbf{e}}+\mathbf{K}_{P} \mathbf{e}
$$

Here $\hat{\mathbf{N}}(\mathbf{q}, \dot{\mathbf{q}}, \ddot{\mathbf{q}})$ can be estimated by the TDE [8-10], as

$$
\hat{\mathbf{N}}(\mathbf{q}, \dot{\mathbf{q}}, \ddot{\mathbf{q}})=\mathbf{N}(\mathbf{q}, \dot{\mathbf{q}}, \ddot{\mathbf{q}})_{t-L},
$$

where $\hat{\mathbf{N}}(\mathbf{q}, \dot{\mathbf{q}}, \ddot{\mathbf{q}})$ denotes the estimate of $\mathbf{N}(\mathbf{q}, \dot{\mathbf{q}}, \ddot{\mathbf{q}})$, ${ }_{t-L}$ denotes time-delayed value of $\bullet$, and $L$ is the estimation time delay, which is the sampling period in digital implementation.

From (2), we can obtain

$$
\mathbf{N}(\mathbf{q}, \dot{\mathbf{q}}, \ddot{\mathbf{q}})_{t-L}=\boldsymbol{\tau}_{t-L}-\overline{\mathbf{M}} \ddot{\mathbf{q}}_{t-L} .
$$

Thus, with the combination of (5) - (8), the TDC control law for a robot manipulator is expressed by [10]

$$
\boldsymbol{\tau}=\boldsymbol{\tau}_{t-L}-\overline{\mathbf{M}} \ddot{\mathbf{q}}_{t-L}+\overline{\mathbf{M}}\left(\ddot{\mathbf{q}}_{d}+\mathbf{K}_{D} \dot{\mathbf{e}}+\mathbf{K}_{P} \mathbf{e}\right) .
$$

Substituting the control input (9) and (8) into robot dynamics (2) yields the closed-loop dynamics, as

$$
\ddot{\mathbf{e}}+\mathbf{K}_{D} \dot{\mathbf{e}}+\mathbf{K}_{P} \mathbf{e}=\overline{\mathbf{M}}^{-1}\left[\mathbf{N}(\mathbf{q}, \dot{\mathbf{q}}, \ddot{\mathbf{q}})-\mathbf{N}(\mathbf{q}, \dot{\mathbf{q}}, \ddot{\mathbf{q}})_{t-L}\right]
$$

If identity of $\mathbf{N}=\mathbf{N}_{t-L}$ assumed, the closed loop equation is (4), the desired error dynamics. The well-known stability condition for the TDC is established by YoucefToumi and Hsia independently in [31-33], expressed by 


$$
\left\|\mathbf{I}-\mathbf{M}^{-1} \overline{\mathbf{M}}\right\|<1
$$

When the closed loop system is stable using the stability criterion (11), the $\mathbf{N}-\mathbf{N}_{t-L}$ is bounded because $\mathbf{N}$ is sum of continuous terms and bounded discontinuous terms $[18,19]$. The bounded TDE error $\boldsymbol{\varepsilon}$ is defined as

$$
\boldsymbol{\varepsilon} \triangleq \overline{\mathbf{M}}^{-1}\left(\mathbf{N}-\mathbf{N}_{t-L}\right)
$$

Then the closed loop system dynamics with the TDC (9) becomes

$$
\ddot{\mathbf{e}}+\mathbf{K}_{D} \dot{\mathbf{e}}+\mathbf{K}_{P} \mathbf{e}=\boldsymbol{\varepsilon} .
$$

The TDE error $\boldsymbol{\varepsilon}$ is close to 0 in most of operating time of robot manipulators; however, it exhibits a pulse-type error due to discontinuity of Coulomb friction at velocity reversal [20].

\subsection{Proposed control using the TDE and fuzzy logic}

To suppress the effect of the TDE error, $\boldsymbol{\varepsilon}$, we adopted the integral sliding surface (ISS), as

$$
\mathbf{s} \triangleq \int_{0}^{t}\left(\ddot{\mathbf{e}}+\mathbf{K}_{D} \dot{\mathbf{e}}+\mathbf{K}_{P} \mathbf{e}\right) d t
$$

Achieving (4) is equivalent to obtain the integral sliding surface $\mathbf{s}=0$. The control input can be designed as

$$
\boldsymbol{\tau}=\overline{\mathbf{M}} \mathbf{u}+\hat{\mathbf{N}}(\mathbf{q}, \dot{\mathbf{q}}, \ddot{\mathbf{q}})
$$

and

$$
\mathbf{u}=\mathbf{u}_{0}+\mathbf{K} \cdot \mathbf{f u z z y}(\mathbf{s}) .
$$

Then, with the combination of (7), (8), (15), (16), the proposed control law for a robot manipulator is expressed by

$$
\boldsymbol{\tau}=\boldsymbol{\tau}_{t-L}-\overline{\mathbf{M}} \ddot{\mathbf{q}}_{t-L}+\overline{\mathbf{M}}\left[\ddot{\mathbf{q}}_{d}+\mathbf{K}_{D} \dot{\mathbf{e}}+\mathbf{K}_{P} \mathbf{e}+\mathbf{K} \cdot \mathbf{f u z z y}(\mathbf{s})\right] .
$$

In (17), the past acceleration $\ddot{\mathbf{q}}_{t-L}$ is given by numerical differentiation as

$$
\ddot{\mathbf{q}}_{t-L}=\left(\mathbf{q}_{t}-2 \mathbf{q}_{t-L}+\mathbf{q}_{t-2 L}\right) / L^{2} .
$$

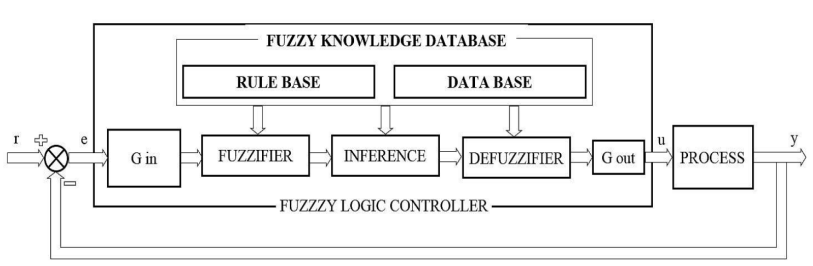

Fig. 1. Fuzzy logic controller

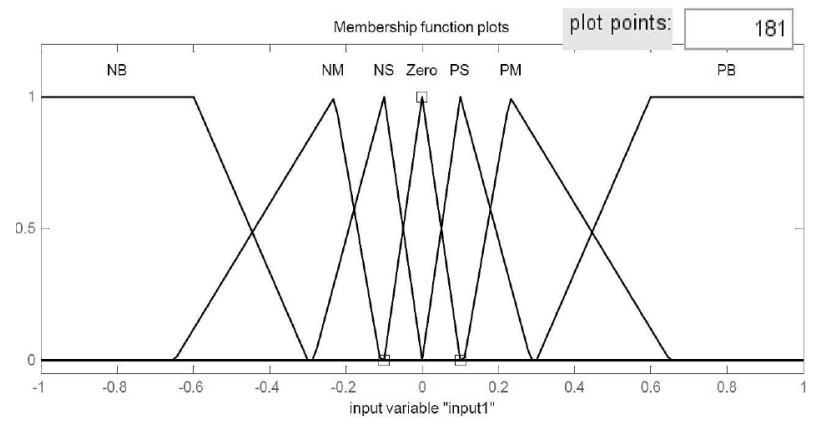

Fig. 2. Fuzzy inference system variables: input

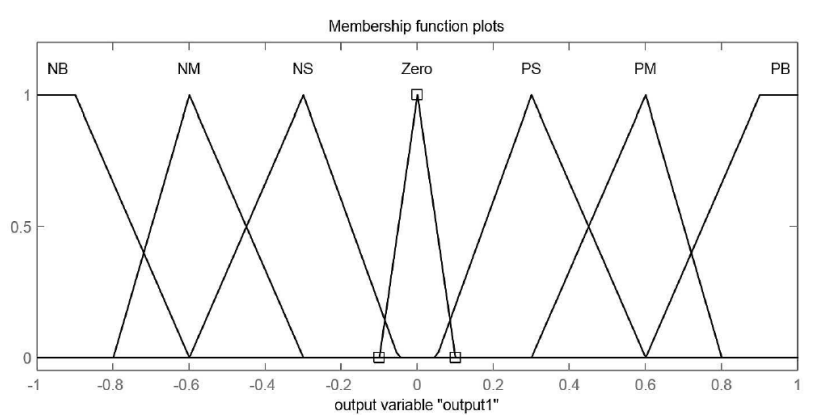

Fig. 3. Fuzzy inference system variables: output

In the proposed control, (17), $\boldsymbol{\tau}_{t-L}-\overline{\mathbf{M}} \ddot{\mathbf{q}}_{t-L}$ stands for the TDE part, $\overline{\mathbf{M}}\left(\ddot{\mathbf{q}}_{d}+\mathbf{K}_{D} \dot{\mathbf{e}}+\mathbf{K}_{P} \mathbf{e}\right)$ injects the desired error dynamics, and $\mathbf{K} \cdot \mathbf{f u z z y}(\mathbf{s})$ corrects the TDE error with ISS (14).

Substituting the control input (17), (14) into robot dynamics (2) yields the closed loop dynamics

$$
\mathbf{u}_{0}-\ddot{\mathbf{q}}+\mathbf{K} \cdot \mathbf{f u z z y}(\mathbf{s})=\boldsymbol{\varepsilon}
$$

or

$$
\dot{\mathbf{s}}+\mathbf{K} \cdot \mathbf{f u z z y}(\mathbf{s})=\boldsymbol{\varepsilon} .
$$

If the fuzzy s-dynamics (21) is asymptotically stable, and $\boldsymbol{\varepsilon}$ is bounded, then the close loop dynamics is bounded. Proof of boundedness of $\boldsymbol{\varepsilon}$ is given in the Appendix.

$$
\dot{\mathbf{s}}+\mathbf{K} \cdot \mathbf{f u z z y}(\mathbf{s})=\mathbf{0} .
$$

Generally, whether a fuzzy control design, shown in Fig. 1 , will be stable is a somewhat open question. Structure of a fuzzy controller is determined by the components such as fuzzy sets, fuzzy rules, fuzzy logic operators, the fuzzy inference method, and the defuzzifier. The structure of a fuzzy controller will be different if different types of the components are used. There are various ways to design an asymptotically stable fuzzy logic controller $[34,35]$. In this paper, we used a Mamdani-type fuzzy inference system (FIS) to construct an asymptotically stable fuzzy 
s-dynamics (21). The Matlab fuzzy logic toolbox is used to design the FIS intuitively.

The fuzzy rules are as follows:

Rule1: If input is Zero then output is Zero.

Rule2: If input is negative big (NB) then output is NB.

Rule3: If input is negative medium (NM) then output is NM.

Rule4: If input is negative small (NS) then output is NS.

Rule5: If input is positive big (PB) then output is $\mathrm{PB}$.

Rule6: If input is positive small (PM) then output is PM.

Rule7: If input is positive small (PS) then output is PS.

Input/output functions are mapped into fuzzy numbers using triangular and trapezoidal membership functions (Figs. 2, 3). In Fig. 2, the width of the Zero set membership function is relatively narrow compared with other membership functions, in order to increase control accuracy in the vicinity of the Zero. To obtain the fuzzy surface, singleton fuzzifier minimum inference engine and centroid defuzzifier are used.

Triangle-shaped membership function and trapezoidalshaped membership function are used for input and output membership functions for the FIS, given by

$$
\begin{gathered}
f(x ; a, b, c)=\left\{\begin{array}{cc}
0, & x \leq a \\
(x-a) /(b-a), & a \leq x \leq b \\
(c-x) /(c-b), & b \leq x \leq c \\
0, & c \leq x
\end{array}\right. \text { and } \\
f(x ; a, b, c, d)=\left\{\begin{array}{cc}
0, & x \leq a \\
(x-a) /(b-a), & a \leq x \leq b \\
1, & b \leq x \leq c \\
(d-x) /(d-c), & c \leq x \leq d \\
0, & d \leq x
\end{array}\right. \text { respectively. }
\end{gathered}
$$

And the input membership functions in Fig. 2 are expressed as follows:

$$
\begin{aligned}
& \mu_{N B}(x)=f(x ;-1.5,-1,-0.6,-0.3), \\
& \mu_{N M}(x)=f(x ;-0.65,-0.23,-0.11), \\
& \mu_{N S}(x)=f(x ;-0.285,-0.1,0), \\
& \mu_{\text {ZERO }}(x)=f(x ;-0.1,0,0.1), \\
& \mu_{P S}(x)=f(x ; 0,0.1,0.285), \\
& \mu_{P M}(x)=f(x ; 0.11,0.23,0.65), \\
& \mu_{P B}(x)=f(x ; 0.3,0.6,1,1.5) .
\end{aligned}
$$

The output membership functions in Fig. 3 are expressed as follows:

$$
\begin{aligned}
& \mu_{N B}(x)=f(x ;-1.5,-1,-0.9,-0.6), \\
& \mu_{N M}(x)=f(x ;-0.8,-0.6,-0.3), \\
& \mu_{N S}(x)=f(x ;-0.6,-0.3,-0.05), \\
& \mu_{\text {ZERO }}(x)=f(x ;-0.1,0,0.1),
\end{aligned}
$$

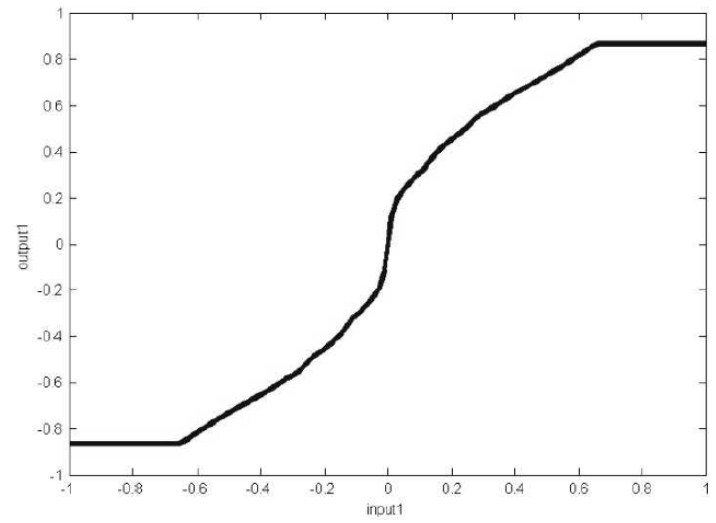

Fig. 4. Control surface of the fuzzy inference system

$$
\begin{aligned}
& \mu_{P S}(x)=f(x ; 0.05,0.3,0.6), \\
& \mu_{P M}(x)=f(x ; 0.3,0.6,0.8), \\
& \mu_{P B}(x)=f(x ; 0.6,0.9,1,1.5) .
\end{aligned}
$$

The control surface of the FIS, depicted in Fig. 4, is an odd function because of the symmetric property of the input/output functions and the use of centroid defuzzifier. Generally, a control surface of FIS is not always an odd function, however, one can carefully select the parameters of input/output membership function to make the control surface function be an odd function; thus, it becomes $s_{i} \cdot \operatorname{fuzzy}\left(s_{i}\right)>0$. If we consider a Lyapunov function as $V=0.5 \mathbf{s}^{T} \mathbf{s}$, then $\dot{V}=\sum \dot{s}_{i} s_{i}=-\sum s_{i} \cdot k_{i} \operatorname{fuzzy}\left(s_{i}\right)<0$, which assures asymptotic stability of the fuzzy s-dynamics (21). The convergent speed is faster in the vicinity of the zero than in the distance of the zero.

\section{Experimental Studies}

A PUMA-type robot, Samsung Faraman AT2 ${ }^{\circledR}$, was used for the experiments (Fig. 5). The maximum payload of the robot is $3 \mathrm{~kg}$. The maximum continuous torques are 0.637 , 0.637 , and $0.319 \mathrm{Nm}$ for joints 1,2 , and 3, respectively. The gear reduction ratio of each joint is $120: 1$, the encoder

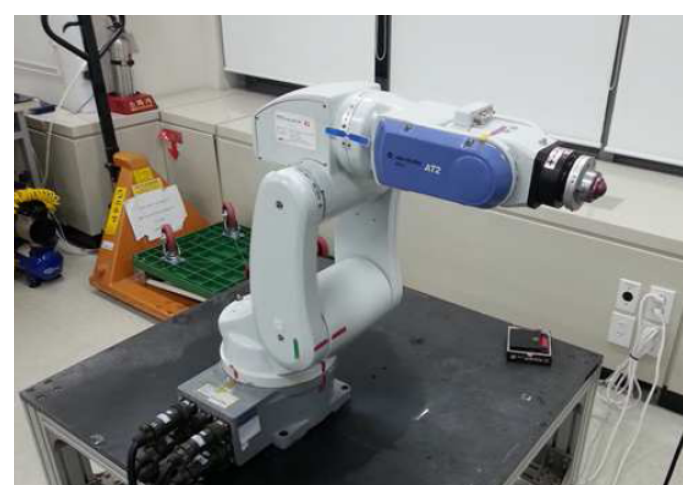

Fig. 5. A PUMA-type robot manipulator 
resolution of each joint is 8192 pulses/rev. Resolution of each robot joint is $3.66 \times 10^{-4} \mathrm{deg}$.

The control objective is to rotate each joint from the initial position $\left(0^{\circ}\right)$ to the first desired position $\left(30^{\circ}\right)$, and then return to the initial position for $0 \mathrm{~s} \leq t<10 \mathrm{~s}$; this process is repeated for the second desired position $10 \mathrm{~s} \leq t<20 \mathrm{~s}$. The desired trajectory is generated using the fifth polynomial method (Fig. 6). The sampling time is given as $L=2 \mathrm{~ms}$.

The desired error dynamics was chosen as $\ddot{\mathbf{e}}+20 \dot{\mathbf{e}}+100 \mathbf{e}=\mathbf{0}$; thus, $k_{D}=20$, and $k_{P}=100$ for each joint. The gain $\overline{\mathbf{M}}=\operatorname{diag}(0.789,0.713,0.357) \mathrm{Kg} \cdot \mathrm{m}^{2}$ for joints 1,2 , and 3 .

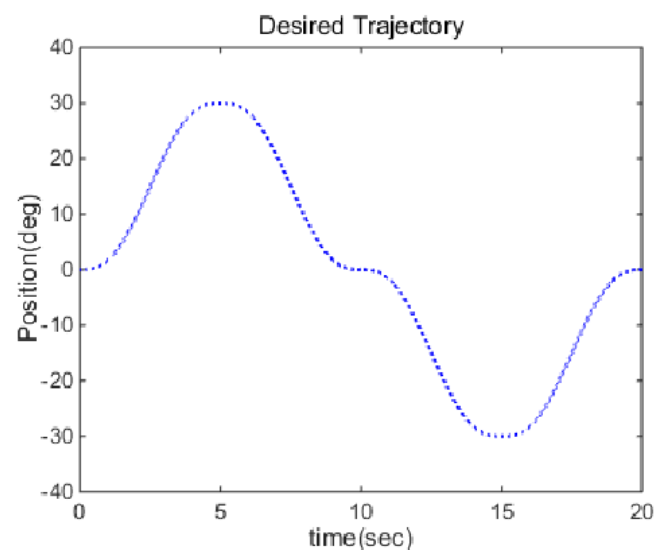

Fig. 6. Desired trajectory for experiments
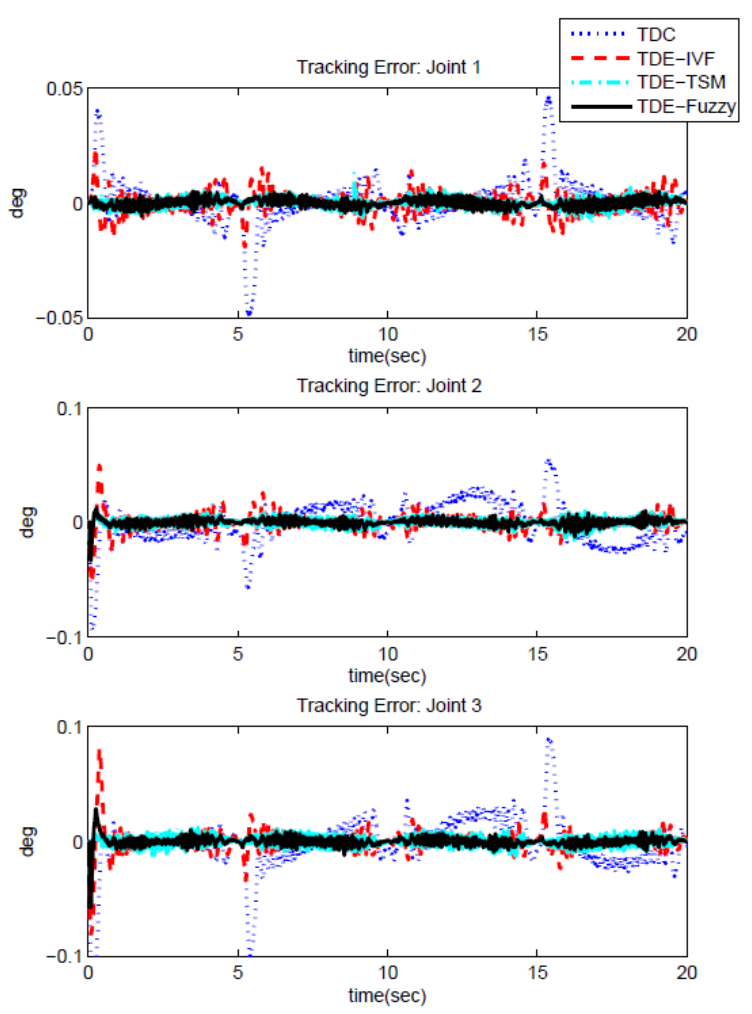

Fig. 7. Comparison of tracking errors
The proposed controller (TDE-Fuzzy) has been compared with the Hsia's TDC control law (9) [10], the TDE-IVF (22) [19], and the TDE-TSM (23) [22].

$$
\begin{aligned}
& \boldsymbol{\tau}=\boldsymbol{\tau}_{t-L}-\overline{\mathbf{M}} \ddot{\mathbf{q}}_{t-L}+\overline{\mathbf{M}}\left(\ddot{\mathbf{q}}_{d}+\mathbf{K}_{D} \dot{\mathbf{e}}+\mathbf{K}_{P} \mathbf{e}+\mathbf{K} \cdot \mathbf{s}\right) . \\
& \boldsymbol{\tau}=\boldsymbol{\tau}_{t-L}-\overline{\mathbf{M}} \ddot{\mathbf{q}}_{t-L}+\overline{\mathbf{M}}\left[\ddot{\mathbf{q}}_{d}+\mathbf{K}_{D} \dot{\mathbf{e}}+\mathbf{K}_{P} \mathbf{e}+\mathbf{K} \cdot \operatorname{sig}(\mathbf{s})^{\gamma}\right]
\end{aligned}
$$

The additional gains for the TDE-IVF and the TDE-TSM are $\mathbf{K}=\operatorname{diag}(10.0,15 . .0,20.0)$ and $\gamma=\operatorname{diag}(0.4,0.4,0.4)$.

Fig. 7 shows the tracking errors of the TDC, TDC-IVF, TDE-TSM, and the proposed TDC-Fuzzy controller. The proposed controller shows the smallest size of tracking error among the four controllers. Using a carefully designed fuzzy logic controller to suppress the effect of the TDE error can improve the tracking performance. In Fig. 8, the proposed controller shows the smallest magnitude of strajectories among the four controllers, showing good agreement with the tendency of tracking errors shown in Fig. 7. Because tracking error $\boldsymbol{e}$ is a filtered version of $\boldsymbol{s}$, bounds on $s$ can be directly translated into bounds on the tracking error. The experimental results confirm that the best tracking performance, as well as s-trajectory tracking, can be achieved through the proposed fuzzy logic based controller. The control input of TDE-TSM (when $0<\gamma<1$ ) shows larger chattering compared with the proposed controller (Fig. 9).

To explain the superiority of the proposed controller to the TDC-IVF and the TDE-TSM, graphical comparisons
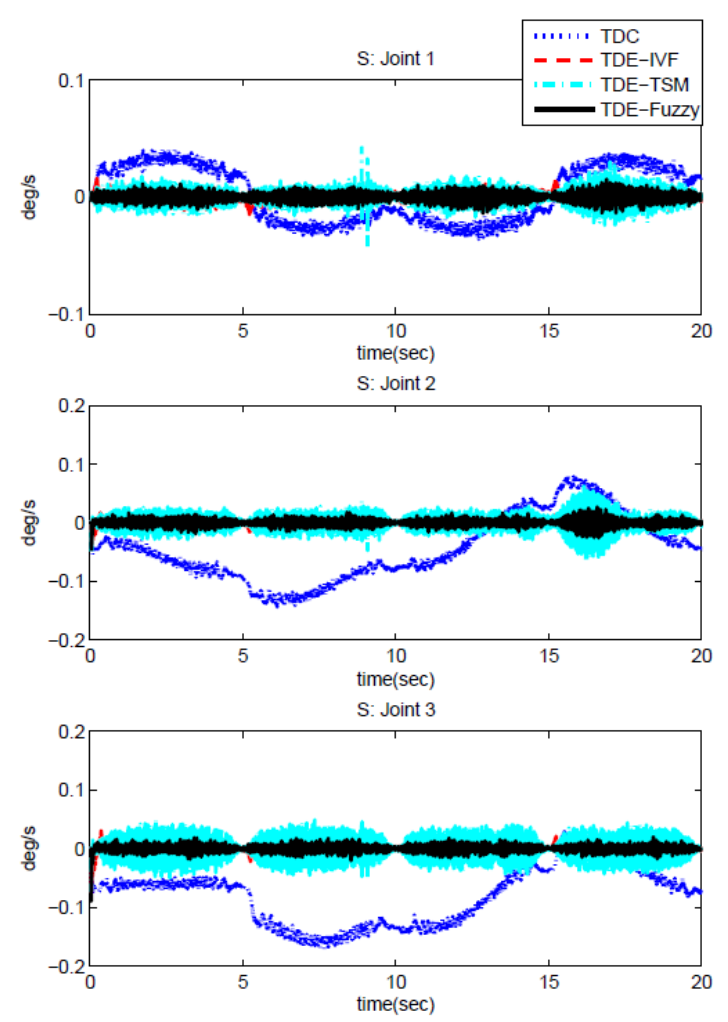

Fig. 8. Sliding variables 

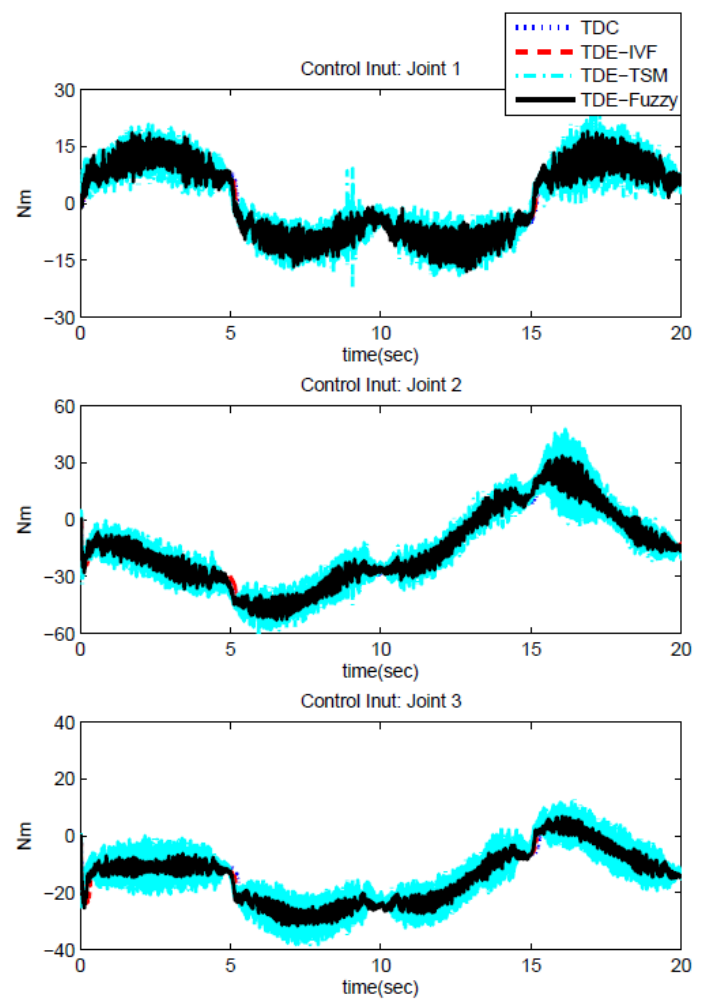

Fig. 9. Control Inputs

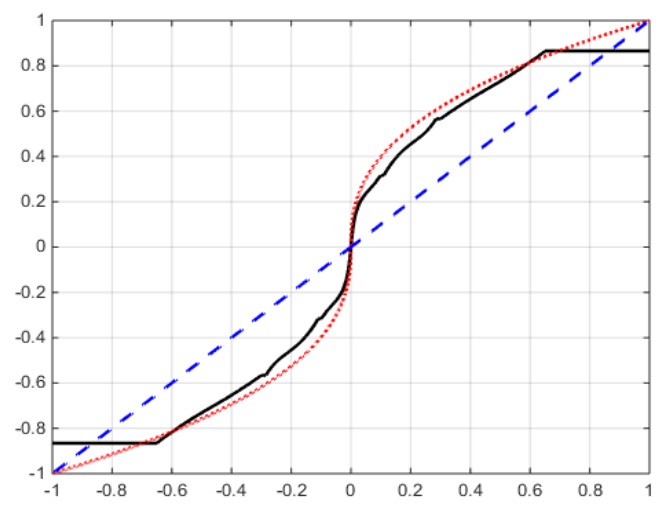

Fig. 10. Graphical comparison of functions: FIS function (black solid), $\operatorname{sig}(s)^{0.4}$ (red dot), and $s$ (blue dashed)

of the FIS function, the TSM function, and the linear functions are conducted in Fig. 10 and Fig. 11. The slope of the FIS function and the TSM function are increasing near the equilibrium point, while the slope of the linear function remains constant. Therefore, the tracking error of the TDE-TSM and TDE-fuzzy is smaller than the TDCIVF.

The slope of the TSM function near the 0 is almost 90 degree, and its shape is almost like signum function which can possibly cause high frequency control chattering (Fig 11). It is well known that the control input chattering harms the tracking performance. In contrast, the slope of the FIS is much smaller than $90^{\circ}$ (Fig 11); thus, chattering

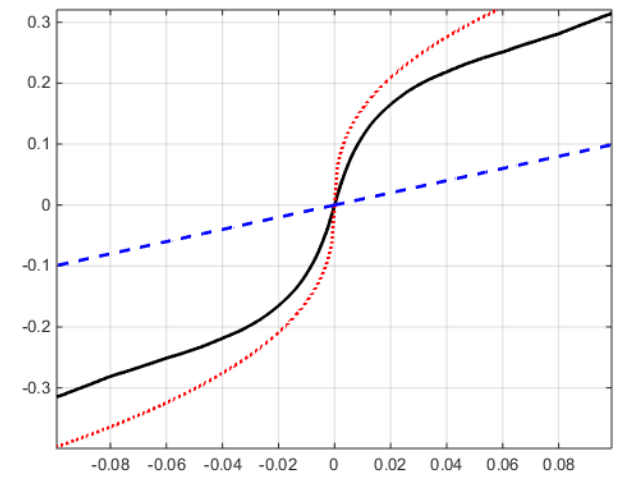

Fig. 11. Magnified graphical comparison of functions: FIS function (black solid), $\operatorname{sig}(s)^{0.4}$ (red dot), and $s$ (blue dashed)

is reduced in the proposed controller. Consequently, the tracking performance of the proposed controller is better than that of the TDE-TSM with less control input chattering.

\section{Conclusion}

In this paper, fuzzy logic is first combined with the TDE technique to suppress the TDE. The proposed controller consists of three parts: the TDE part to estimate and cancel continuous nonlinearities of robot dynamics, the injection part to endow desired dynamics, and the correcting part using integral sliding mode and Mamdani-type FIS. The Mamdani-type FIS is constructed by using common sense rules that refer to indefinite quantities. The proposed controller with well-designed FIS functions is highly intuitive, efficient, and accurate. Thanks to the TDE part, the proposed controller preserves the simplicity and efficiency of TDC. Compared with the TDE-TSM control, better tracking performance is achieved using the proposed controller, while control chattering is reduced significantly. The proposed controller, with the help of FLC, could be a good replacement of existing TDE based controllers to achieve good tracking performance and chattering reduction. Fuzzy Logic Systems used in this paper is simple and standard. One can expect that more sophisticated intelligent systems (such as artificial neural networks (ANN), fuzzy $\mathrm{ANN}$, and neuro-fuzzy systems) can further enhance the control performance of the proposed controller. More researches in the near future, based on this framework, are welcome.

\section{Acknowledgements}

This work was supported by the Ministry of Trade, Industry \& Energy (MOTIE, Korea) under Industrial Technology Innovation Program. No.10067184, "Development of armored robot systems for personal protections of 
rescuemen and emergency management operations in the composite disaster site".

\section{References}

[1] G. Zidani, S. Drid, L. Chrifi-Alaoui, D. Arar, and P. Bussy, "Robust nonlinear control of a mobile robot," JEET, vol. 11, no. 4, pp. 1012-1019, 2016.

[2] C. Urrea and J. Kern, "Trajectory tracking control of a real redundant manipulator of the SCARA type," JEET, vol. 11, no. 3, pp. 751-758, 2016.

[3] F. L. Lewis, C. T. Abdallah, and D. M. Dawson, Control of robot manipulators. New York: Macmillan, 1993.

[4] M. Uebel, I. Minis, and K. Cleary, "Improved computed torque control for industrial robots," in Proc. IEEE Int. Conf. Robot. Autom., vol. 1, 1992, pp. 528-553.

[5] J. Slotine, "The robust control of robot manipulators," Int. J. Robotics Research, vol. 4, no. 2, pp. 49-64, 1985.

[6] R. Morgan and U. Ozguner, "A decentralized variable structure control algorithm for robotic manipulators," IEEE Trans. Robot. Autom., vol. RA-1, pp. 57-65, Mar. 1985.

[7] K. Youcef-Toumi and O. Ito, "Controller design for systems with unknown dynamics," in American Control Conference, vol. 2, 1987, pp. 836-844.

[8] T. C. Hsia, "A new technique for robust control of servo systems," IEEE Trans. Ind. Electron., vol. 36, no. 1, pp. 1-7, Feb. 1989.

[9] K. Youcef-Toumi and O. Ito, "A time delay controller for systems with unknown dynamics," Trans. ASME J. Dyn. Syst. Meas. Control, vol. 112, no. 1, pp. 133142, 1990.

[10] T. S. Hsia, T. Lasky, and Z. Guo, "Robust independent joint controller design for industrial robot manipulators," IEEE Trans. Ind. Electron., vol. 38, no. 1, pp. 21-25, 1991.

[11] K. H. Kim, H. S. Kim, and M. J. Youn, "An improved stationary-framebased current control scheme for a permanent-magnet synchronous motor," IEEE Trans. Ind. Electron., vol. 50, no. 5, pp. 1065-1068, May 2003.

[12] Y. X. Wang, D. H. Yu, and Y. B. Kim, "Robust timedelay control for the DC-DC boost converter," IEEE Trans. Ind. Electron., vol. 61, no. 9, pp. 4829-4837, Sep. 2014.

[13] M. Jin, J. Lee, and K. K. Ahn, "Continuous nonsingular terminal sliding-mode control of shape memory alloy actuators using time delay estimation," IEEE/ASME Trans. Mechatronics, vol. 20, no. 2, pp. 899-909, Apr. 2015.

[14] J. Lee, M. Jin, and K. K. Ahn, "Precise tracking control of shape memory alloy actuator systems using hyperbolic tangential sliding mode control with time delay estimation," Mechatronics, vol. 23, no. 3, pp. 310-317, 2013.

[15] M. Jin and P. H. Chang, "Simple robust technique using time delay estimation for the control and synchronization of lorenz systems," Chaos, Solitons \& Fractals, vol. 41, no. 5, pp. 2672-2680, 2009.

[16] J. Kim and M. Jin, "Synchronization of chaotic systems using particle swarm optimization and timedelay estimation," Nonlinear Dynamics, vol. 86, no. 3, pp. 2003-2015, Nov. 2016.

[17] T. C. Hsia and S. Jung, "A simple alternative to neural-network control scheme for robot manipulators," IEEE Trans. Ind. Electron., vol. 42, no. 4, pp. 414416, 1995.

[18] S. Jung, T. Hsia, and R. Bonitz, "Force tracking impedance control of robot manipulators under unknown environment," IEEE Trans. Control Syst. Technol., vol. 12, no. 3, pp. 474-483, May 2004.

[19] M. Jin, S. H. Kang, and P. H. Chang, "Robust compliant motion control of robot with nonlinear friction using time-delay estimation," IEEE Trans. Ind. Electron., vol. 55, no. 1, pp. 258-269, 2008.

[20] M. Jin, J. Lee, P. H. Chang, and C. Choi, "Practical nonsingular terminal sliding-mode control of robot manipulators for high-accuracy tracking control," IEEE Trans. Ind. Electron., vol. 56, no. 9, pp. 14061414, Sep. 2009.

[21] J. Lee, P. H. Chang, and R. S. Jamisola, "Relative impedance control for dual-arm robots performing asymmetric bimanual tasks," IEEE Trans. Ind. Electron., vol. 61, no. 7, pp. 3786-3796, Jul. 2014.

[22] M. Jin, Y. Jin, P. H. Chang, and C. Choi, "Highaccuracy tracking control of robot manipulators using time delay estimation and terminal sliding mode," Int J. Adv. Robot. Syst., vol. 8, no. 4, pp. 65-78, Sep. 2011.

[23] Very slow std::pow() for bases very close to 1, http://stackoverflow.com/questions/14687665/veryslow-stdpow-for-bases-very-close-to-1

[24] Slow power computation by 64-bit glibc, http://entropymine.com/imageworsener/slowpow/

[25] Optimized approximative pow() in c / c++, http://martin.ankerl.com/2012/01/25/optimized approximative-pow-in-c-and-cpp/,

[26] Fast implementation/approximation of pow() function, http://stackoverflow.com/questions/2347138/fastimplementation-approximation-of-pow-function-in-c-c

[27] Fast approximate logarithm, exponential, power, http://www.machinedlearnings.com/2011/06/fastappr oximate-logarithm-exponential.html

[28] E. H. Mamdani, "Application of fuzzy logic to approximate reasoning using linguistic synthesis," IEEE Trans. Computers, vol. C-26, no. 12, pp. 11821191, Dec 1977.

[29] J. Mendel, "Fuzzy logic systems for engineering: a 
tutorial," IEEE Proceedings, vol. 83, no. 3, pp. 345377, Mar 1995.

[30] Q. Yu, Y. Shi, M. Cai, and W. Xu, "Fuzzy logic speed control for the engine of an air-powered vehicle," Advances in Mechanical Engineering, vol. 8, no. 3, 2016.

[31] T. C. Hsia and L. S. Gao, "Robot manipulator control using decentralized linear time-invariant time-delayed joint controllers," in Proc. IEEE int. Conf. Robot. Autom., May 1990, pp. 2070-2075.

[32] K. Youcef-Toumi and S.-T. Wu, "Input / output linearization using time delay control," Trans. ASME J. Dyn. Syst. Meas. Control, vol. 114, no. 1, pp. 10-19, 1992.

[33] M. Jin, J. Lee, and N. G. Tsagarakis, "Model-free robust adaptive control of humanoid robots with flexible joints," IEEE Trans. Ind. Electron., vol. 64, no. 2, pp. 1706-1715, Feb. 2017.

[34] H. Ying, "Structure and stability analysis of general mamdani fuzzy dynamic models," Int. J. Intelligent Syst., vol. 20, no. 1, pp. 103-125, 2005.

[35] M. Margaliot and G. Langholz, "Fuzzy Lyapunovbased approach to the design of fuzzy controllers," Fuzzy Sets and Systems, vol. 106, no. 1, pp. 49-59, 1999.

\section{Appendix: Stability}

The boundedness of $\boldsymbol{\varepsilon}$ can be proved in the same manner of the stability proof in [18-20].

Using (16), (19) gives

$$
\boldsymbol{\varepsilon}=\mathbf{u}-\ddot{\mathbf{q}}
$$

A combination of (5), (8), (16), (17), and (24) gives

$$
\begin{aligned}
\mathbf{M \varepsilon} \boldsymbol{\varepsilon} & =\mathbf{M}(\mathbf{u}-\ddot{\mathbf{q}}) \\
& =\mathbf{M u}+\mathbf{C} \dot{\mathbf{q}}+\mathbf{G}+\mathbf{F}+\boldsymbol{\tau}_{d}-\boldsymbol{\tau} \\
& =\mathbf{M u}+\mathbf{C} \dot{\mathbf{q}}+\mathbf{G}+\mathbf{F}+\boldsymbol{\tau}_{d}-\overline{\mathbf{M}} \mathbf{u}-\mathbf{N}_{t-L} .
\end{aligned}
$$

From (3), the delayed nonlinear term is given by

$$
\begin{aligned}
\mathbf{N}_{t-L}= & {\left[\mathbf{M}_{t-L}-\overline{\mathbf{M}}\right] \ddot{\mathbf{q}}_{t-L} } \\
& +(\mathbf{C} \dot{\mathbf{q}})_{t-L}+\mathbf{G}_{t-L}+\mathbf{F}_{t-L}+\left(\boldsymbol{\tau}_{d}\right)_{t-L}
\end{aligned}
$$

Substitute (26) into(25), we have

$$
\mathbf{M} \boldsymbol{\varepsilon}=(\mathbf{M}-\overline{\mathbf{M}}) \mathbf{u}-\left(\mathbf{M}_{t-L}-\overline{\mathbf{M}}\right) \ddot{\mathbf{q}}_{t-L}+\Delta,
$$

where

$$
\Delta=\mathbf{C} \dot{\mathbf{q}}+\mathbf{G}+\mathbf{F}+\boldsymbol{\tau}_{d}-(\mathbf{C} \dot{\mathbf{q}})_{t-L}-\mathbf{G}_{t-L}-\mathbf{F}_{t-L}-\left(\boldsymbol{\tau}_{d}\right)_{t-L}
$$

It is clear that $\boldsymbol{\Delta}$ is bounded for a sufficiently small $L$. Substituting $\ddot{\mathbf{q}}_{t-L}=\mathbf{u}_{t-L}-\boldsymbol{\varepsilon}_{t-L}$ from (24) yields

$$
\begin{aligned}
\mathbf{M \varepsilon}= & (\mathbf{M}-\overline{\mathbf{M}}) \mathbf{u}-(\mathbf{M}-\overline{\mathbf{M}}) \ddot{\mathbf{q}}_{t-L} \\
& +\left(\mathbf{M}-\mathbf{M}_{t-L}\right) \ddot{\mathbf{q}}_{t-L}+\Delta \\
= & (\mathbf{M}-\overline{\mathbf{M}}) \mathbf{u}-(\mathbf{M}-\overline{\mathbf{M}})\left(\mathbf{u}_{t-L}-\boldsymbol{\varepsilon}_{t-L}\right) \\
& +\left(\mathbf{M}-\mathbf{M}_{t-L}\right) \ddot{\mathbf{q}}_{t-L}+\Delta \\
= & (\mathbf{M}-\overline{\mathbf{M}}) \boldsymbol{\varepsilon}_{t-L}+(\mathbf{M}-\overline{\mathbf{M}})\left(\mathbf{u}-\mathbf{u}_{t-L}\right) \\
& +\left(\mathbf{M}-\mathbf{M}_{t-L}\right) \ddot{\mathbf{q}}_{t-L}+\Delta
\end{aligned}
$$

Therefore, $\boldsymbol{\varepsilon}$ is given by

$$
\boldsymbol{\varepsilon}=\mathbf{E} \boldsymbol{\varepsilon}_{t-L}+\mathbf{E} \boldsymbol{\eta}_{1}+\boldsymbol{\eta}_{2},
$$

where

$$
\begin{gathered}
\mathbf{E}=\mathbf{I}-\mathbf{M}^{-1} \overline{\mathbf{M}} \\
\mathbf{E}=\mathbf{I}-\mathbf{M}^{-1} \overline{\mathbf{M}} \boldsymbol{\eta}_{1}=\mathbf{u}-\mathbf{u}_{t-L} \\
\boldsymbol{\eta}_{2}=\mathbf{M}^{-1}\left[\left(\mathbf{M}-\mathbf{M}_{t-L}\right) \ddot{\mathbf{q}}_{t-L}+\Delta\right] .
\end{gathered}
$$

For a sufficiently small time delay $L, \boldsymbol{\eta}_{1}$, and $\boldsymbol{\eta}_{2}$ are bounded.

In the discrete-time domain (30) can be represented as

$$
\boldsymbol{\varepsilon}(k)=\mathbf{E}(k) \boldsymbol{\varepsilon}(k-1)+\mathbf{E}(k) \boldsymbol{\eta}_{1}(k)+\boldsymbol{\eta}_{2}(k) .
$$

Therefore, if $\|\mathbf{E}\|<1$, then roots of $\mathbf{E}(k)$ reside inside a unit circle, and (34) is asymptotically bounded with bounded forcing function $\boldsymbol{\eta}_{1}$, and $\boldsymbol{\eta}_{2}$.

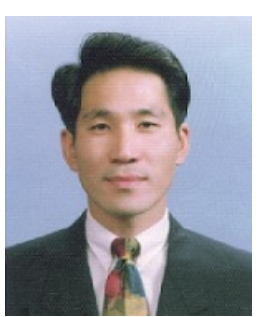

Hyo-Jeong Bae He received the B.S. degree in mechanical design from Kyungil University in 1997, and the M.S. degree in intelligent mechanical engineering from Pukyong National University in 2004. He is currently working toward the Ph.D. degree in Pukyong National University. Since 1992, he has been with the Robotics and Engineering Solutions Research Group, Research Institute of Industrial Science \& Technology (RIST), Korea. His research interests include robust control of nonlinear MIMO systems, timedelay control, robot motion control, web-based remote control, intelligent automation of large mobile devices, and smart factory.

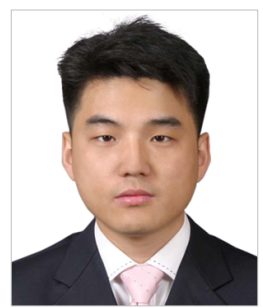

Maolin Jin $\mathrm{He}$ received the B.S. degree in material science and mechanical engineering from Yanbian University of Science and Technology, Jilin, China, in 1999, and the M.S. and Ph.D. degrees in mechanical engineering from Korea Advanced Institute of Science and Technology (KAIST), 
Daejeon, Korea, in 2004 and 2008, respectively. In 2008, he was a Postdoctoral Researcher with the Mechanical Engineering Research Institute, KAIST. From November 2008 to February 2016, he was a Senior Researcher with the Research Institute of Industrial Science and Technology (RIST), Pohang, South Korea. He is currently a Principal Researcher with the Korea Institute of Robots and Convergence (KIRO), Pohang, South Korea. His research interests include robust control of nonlinear plants, timedelay control, robot motion control, electro-hydraulic actuators, winding machines, disaster robotics, and factory automation.

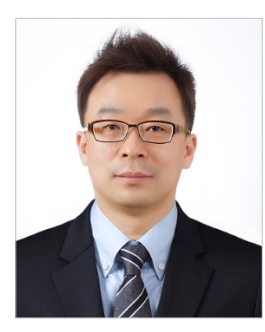

Jinho Suh He received the B.S degree in Mathematics from Hanyang University, and the M.S degree in Mechanical, Environment and Ocean Engineering, from Pukyong National University. He received the $\mathrm{Ph}$. D. from Dept. of Control Engineering, Tokyo Institute of Technology. Dr. Suh was a lecturer with the Dept. of Mechanical Engineering, Pukyong National University. $\mathrm{He}$ is a Director with Disaster Robotics R\&D Center, Korea Institute of Robot \& Convergence (KIRO). He is also an Adjunct Professor with Dept. of Mechanical Engineering, Pohang University of Science and Technology (POSTECH), Korea. His research interests include robust control, system integration, field robotics, and disaster robotics.

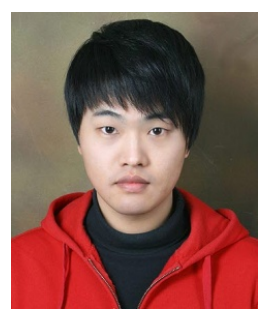

Jun Young Lee He received the B.S. degree in electronic engineering from Keimyung University, Daegu, South Korea, in 2011, and M.S. degrees in robotics engineering from DaeguGyeongbuk Institute of Science and Technology (DGIST), Daegu, South Korea, in 2013, where he is currently working toward the Ph.D. degree. His research interests include robust control of nonlinear plants, robot motion control, and rehabilitation robotics.

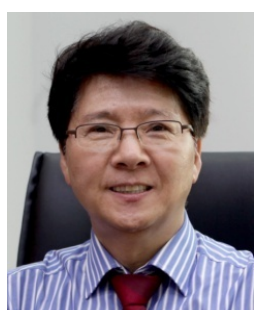

Pyung-Hun Chang He received the B.S. and M.S. degrees in mechanical engineering from Seoul National University, Seoul, Korea, in 1974 and 1977, respectively, and the M.Sc. the Ph.D. degrees in mechanical engineering from the Massachusetts Institute of Technology (MIT), Cambridge, MA, in 1984 and 1987, respectively. He had been a Professor at Korea Advanced Institute of Science and Technology (KAIST), Daejeon, Korea, for 24 years, and now is a
Professor with the Dept. of Robotics Engineering, DaeguGyeongbuk Institute of Science and Technology (DGIST), Daegu, Korea, where he is also the vice president for academic affairs. His current research interests include the rehabilitation robotics, robust control of nonlinear systems including redundant manipulators, and impedance control of dual-arm manipulators.

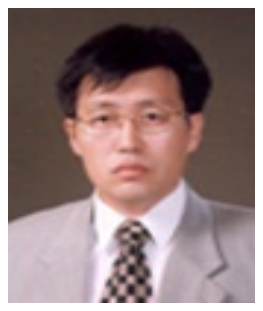

Doo-Sung Ahn He received the B.S degree in mechanical engineering from Seoul National University in 1978 and the M.S. and Ph.D. degrees in precision engineering from Korea Advanced Institute of Science and Technology (KAIST), in 1980 and 1992, respectively. He is a professor with the Dept. of Mechanical Design Engineering, Pukyong National University. His research interests include intelligent control, hydraulic manipulators, and biped dancing robots. 